Journal of Computer Science (Special Issue): 96-98, 2005

ISSN 1549-3636

(C) 2005 Science Publications

\title{
A New Model for Multi-agent Case-base Reasoning Systems
}

\author{
${ }^{1}$ Abdel Badeea Salem, ${ }^{2}$ aldahoud Ali and ${ }^{3}$ Ibrahiem M. M. El Emary \\ ${ }^{1}$ Faculty of Computer and Information Science, Ain Shams University, Cairo, Egypt \\ ${ }^{2}$ Faculty of Information Technology, Al Zaytoonah University, Amman, Jordan \\ ${ }^{3}$ Faculty of Engineering, Amman Al Ahliyya University, Amman, Jordan
}

\begin{abstract}
Multi-Agent Systems (MASs) and Case-Based Reasoning (CBR) are two recent and hot paradigms in artificial intelligence field. CBR is a reasoning methodology based on old experience reasoning or similarity-based reasoning while MAS is a new paradigm to organize AI applications. CBR offers multi-agent systems the capability of autonomously learning from experience. This study examines the integration of CBR, MASs and Expert Systems (ESs). In addition, it presents a knowledge-based model of multi-agent CBR systems from both a logical and a knowledge-based viewpoint.
\end{abstract}

Key words: Multi-agent systems, case-based reasoning systems, multi-agent CBR system

\section{INTRODUCTION}

Case-based reasoning (CBR) and multi-agent systems (MASs) are two different paradigms in AI. $\mathrm{CBR}$ is a reasoning paradigm based on experiencebased reasoning. MAS is a new paradigm to organize AI applications. The integration of CBR and MASs has drawn increasing attention in the AI community because CBR offers MAS paradigm the capability of autonomously learning from experience[1]. This study will fill this gap by providing knowledge-based models of multi-agent CBR systems (CBRSs).

ESs and CBR: This section will examine the relationship between expert systems (ESs) and case based reasoning systems (CBRSs).

Expert Systems: Expert knowledge is a combination of a theoretical understanding of the problem and a collection of heuristic problem-solving rules that experience has shown to be effective in the domain. ESs is constructed by obtaining this knowledge from human experts and coding it into a form that a computer may apply to similar problems.

Early research of ESs arose in universities in the mid-1960s and emphasized matching the performance of human experts. DENDRAL was the first to achieve expert performance and identified the chemical molecular structure of a material from its mass spectrographic and nuclear magnetic resonance. MYCIN used expert medical knowledge to diagnose and prescribe treatment for spinal meningitis and bacterial infections of the blood. ESs used to be one of the success stories of AI research in the 1980s- 1990s. Since this time, Thousands of ESs have been developed and deployed in industrial and commercial settings and permeated nearly every area of industry and government such as finance and management. Generally speaking, ESs has made a significant progress in the following aspects: the key role of knowledge in intelligent simulation; effective knowledge representations and more powerful reasoning techniques[2,3].

In what follows, we will focus on rule-based ESs (RBESs), which are an important part of ESs. An ES mainly consists of a knowledge base (KB) and an inference engine (IE). The KB contains the knowledge used by human experts. The IE consists of all the processes that manipulate the $\mathrm{KB}$ to produce information requested by the user. Thus, we can briefly formalize it as

$\mathrm{ES}=\mathrm{KB}+\mathrm{IE}$

The IE applies the knowledge to the solution of actual problems. It is essentially an interpreter of the KB.

However, despite the undoubted success of ESs, developers of these systems have met several problems as:

* Knowledge acquisition is still a difficult process;

* Implementing an ES is a difficult process requiring special experience and skills and often taking many years;

* Once implemented model-based KBS are often slow and are unable to access or mange large volumes of information.

Over the last ten years, Case-based reasoning (CBR) that seems to address the problems identified above has increasingly attracted more and more attention. The next section will turn to examining CBR.

Corresponding Author: Abdel Badeea Salem, Faculty of Computer and Information Science, Ain Shams University, Cairo, Egypt 
Case-based reasoning: $\mathrm{CBR}$ is a reasoning paradigm based on previous experiences or cases, that is, case based reasoning solves new problems by adapting solutions that were used to solve old problems. In other words, CBR is a form of experience-based reasoning. Therefore, the CBR system (CBRS) is an intelligent system based on experience based reasoning, which can be modeled as[4]:

CBRS $=$ Case Base + CBR Engine

Where the case base (CB) is the set of cases, each of which consists of the previous encountered problem and its solution. CBR engine, similar to the inference engine (IC) in ES, is the inference mechanism for performing experience-based reasoning.

AS we know, "Two cars with similar quality features have similar prices" is a popular experience principle. From a logical viewpoint, this is a kind of similarity-based reasoning. In other words, similaritybased reasoning is a special form of experience based reasoning. Therefore, CBR can be considered as a kind of similarity-based reasoning from a logical viewpoint, although it is still a kind of knowledge-based system from a knowledge-based viewpoint. This means that the CBR system (CBRS) can be formalized as

$\mathrm{CBRS}=\mathrm{CB}+\mathrm{CBRE}$

Where $\mathrm{CB}$ denotes the case base in the CBR system. The CBRE is the inference mechanism for performing similarity-based reasoning. This is the essence of any similarity-based reasoning.

Comparison between RBES and CBRS: As we know, a RBES breaks a problem down into a set of individual rules that each solves part of the problem. Rules are combined together to solve a whole problem. However, to create these rules by hand, you have to know how to solve the problem and this task can be extremely complex and time consuming. CBRSs differ basically in that to use them; one does not need to know how to solve a problem, only to recognize if we have solved a similar problem in the past. Based on this idea, if we have solved a similar problem in the past, then we can use CBRS instead of RBES to solve this problem. In other words, if we have not solved a similar problem in the past, we have to use RBES instead of CBRS to try to solve this problem.

Further, there are real difference between CBRS and RBES. For example:

* Partial matching: in CBRSs usually no cases match exactly in all details. Patterns may be used to recognize and store generalizations about cases, but they are not themselves considered to be cases.

* CBRS does not require an explicit domain model and so elicitation becomes a task of gathering case histories.

* Implementation is reduced to identifying significant features that describe a case, an easier task than creating an explicit model.

However, traditional RBESs and CBRSs share the common theoretical foundation: that is, mathematical logic, because IE and CBRE can basically perform deductive reasoning. More specifically, IE performs reasoning based on modus ponens, while CBRE performs similarity-based reasoning based on generalized modus ponens[5,6].

Because generalized modus ponens, similarity based reasoning is a more general form of deductive reasoning. From the knowledge based viewpoint, the case base $\mathrm{CB}$ in the CBRS corresponds to the knowledge base in the ES. Therefore; we conclude that CBRS can be considered as a general form of RBES, or CBRS can be degenerated to RBES if $\mathrm{CB}$ is degenerated to $\mathrm{KB}$ and $\mathrm{CBRE}$ is to IE. This means that CBRS is similar to ES from a knowledge-based viewpoint; that is:

$\mathrm{CBRS}=\mathrm{CB}+\mathrm{CBRE}=\mathrm{ES}=\mathrm{KB}+\mathrm{IE}$

Multi-agent systems: Multi agent systems (MASs) are among the most rapidly growing areas in AI communities with the rapid development of Internet and WWW. An intelligent agent is a set of computational elements that plan and control the execution of jobs, correcting errors and perturbations along the way. Intelligent agents may help private and business user in their search of information and performance of tasks in a networked digital world and improve their decision making in their business activities.

Intelligent agent technology combines AI (reasoning, planning, natural language processing, etc.) and system development techniques (object-oriented programming, scripting languages, human-machine interface, distributed processing, etc,) to produce a new generation of software that can, based on user preferences, perform tasks for users. The following capabilities are often associated with the notion of an intelligent agent: Adaptability, autonomy cooperatively, mobility, proactively, rationality, reasoning capability and sociability.

As to a single intelligent agent, it does not necessarily have all these abilities. It should be tailored to special problem specification. However, it is probably reasonable to say that the intelligence level of an agent can be correlated to the degree to which it implements these abilities. Thus, it is better to think of agents as providing a range, or different levels of intelligence in the MASs, just as different individuals have different intelligence[7].

From the viewpoint of distributed AI, a MAS is a loosely coupled network of Problem solver entities, which are called agents, that work together to find answer to problems that are beyond the individual capabilities or knowledge of each entity. More recently, the term MAS has been given a more general meaning and it is now used for all types systems composed of multiple agents showing the following characteristics:

* Each agent has incomplete capabilities to solve a problem

* There is no global system control over agents

* Data is decentralized

* Computation is asynchronous 
The following four concepts are vitally important to MASs: coordination, cooperation, communication and negotiation[8].

* Coordination is a property of a system of agents performing some activities in a shared environment.

* Cooperation is coordination among nonantagonistic agents and arises as they plan and execute their actions in a coordinated way to achieve their goals.

* Communication forms the basis of the cooperation and is formed from the communication protocols and the resulting communication methods.

* Negotiation means a compromise for both parties and causes a degradation of their results. The overall aim of all negotiation activities is to permit a constructive cooperation from within the group of independently operating agents that have their own goals.

* For a single intelligent agent, these concepts need not be of importance as it could do all the work on its own.

However, their importance becomes evident in the MASs; standards-based mechanisms and means to coordinate, cooperate and communicate with all kinds of agents are at the root of the MASs.

Relations between ES and MAS: The key ideas of RBESs have affected MAS. Two of these concern the development of MASs, that is, RBESs for communications architecture and macro-rules in the form of pattern-directed modules for distributed architecture and systems of cooperating systems, which can be characterized by cooperating agents. Therefore, MAS technology is a further development of ES technology into account cooperation, coordination, communication and negotiation.

Cooperation is essential to MASs. In fact, using cooperating agent within the MAS has at least two attractive features at the abstract level:

* Using a collection of problem-solvers makes it easier to employ divide and conquer strategies in order to solve complex, distributed problems. Each agent only needs to posses the capabilities and resources to solve as individual, local problem.

* The idea of several agents cooperating to solve a problem that none could solve individually is a powerful metaphor for thinking about various ways that individual elements can be combined to solve complex problems.

Using these features, one can overcome the difficulties of cooperation and coordination in ESs. Some ES literature has already paid attention to the cooperation of the ES with other computer systems.

Communication capability has also become one of important techniques for ESs. ESs communicates experts, databases and other computing systems. Just as humans access and interact with these various sources and ES needs to speak to each in its own appropriate language.
ESs communicates with knowledge engineers through structure editors that allow them to access and modify components of the knowledge base easily. ESs communicates with experts through sample dialogues with explanation that elucidate their lines of reasoning and highlight for the expert where to make knowledge base changes. ESs also interacts with other computing systems. For example, ESs incorporates means to access and retrieve information from online databases.

Based on this discussion, MAS technology fosters research and development of ESs. It can thus be asserted that the simulation of human intelligence depends not only on the computerized knowledge and reasoning of human experts, to which ESs have paid much attentions, but also on the coordination, cooperation and communication between an intelligent system and other computing systems, which MASs have emphasized. This is because the human intelligence depends not only on the possession of the knowledge and reasoning methods, but also heavily on the community where the human being lives or works.

\section{CONCLUSION}

This study examined the relationship between ESs, CBR and MASs from both a logical viewpoint and knowledge based viewpoint and proposed models of multi agent CBR system integrating CBR systems and knowledge-based systems (KBSs), which basically cover most attempts that have applied CBR in MASs in a homogenous or heterogeneous setting.

\section{REFERENCES}

1. Agnar, A., 2001. Modeling the knowledge contents of CBR system. Proc. of the Workshop Program at the Fourth Intl. Conf. on Case Based Reasoning. Vancouver, pp: 32-37.

2. Smyth and McKenna, 1999. Building compact competent case-based. Proc. 3rd Intl. Conf. on CBR. Springer, pp: 329-342.

3. Mohamed, A.H. et al., 2005. Case-Fractional-based Diagnostic System (CPDS). To appear in Engineering Application of Artificial Intelligence, 5: 501-509.

4. Aamodt and Plaza, 1994. Case-based reasoning: Foundational Issues, Methodological Variations and System Approaches. IOS Press, 7: 39-59.

5. Macaya, D., J. Melendez and J. Colomer, 2002. Case based approach for supervision. Application to PID Controllers. IFAC 15th Triennial World Congress, Barcelona, Spain.

6. Watson, I., 1997. Applying Case-bases Reasoning Techniques for Enterprise Systems. Morgan Kaufinann Publishers, Inc., USA.

7. Bonzano, A., P. Cunningham and B. Symth, 1997. Using introspective learning to improve retrieval in CBR: A case study in air traffic control. Proc. of the Second Intl. Conf. on Case Base Reasoning ICCBR-97, Providence RI. USA, pp: 291-302.

8. Haney, K. and M.T. Keane, 1996. Learning adaptation rules from a case-bas. Proc. Third European Workshop on Case-Based Reasoning Springer-Verlag, pp: 179-192. 